\title{
Úlcera tuberculosa intestinal como causa de hemorragia digestiva baja masiva exanguinante*
}

\author{
Drs. RICARDO VILLALÓN C. ${ }^{1}$, MARCO RIOSECO V. ${ }^{1}$, SERGIO RUBEL C. ${ }^{1}$, \\ CARLOS LOYOLA R. ${ }^{1}$, Int. GONZALO MASÍA L. ${ }^{2}$ \\ 1 Servicio de Cirugía y de Urgencia. Complejo Asistencial Barros Luco/Trudeau. \\ 2 Interno Facultad de Medicina, Universidad de Chile. \\ Santiago, Chile.
}

\begin{abstract}
Intestinal tuberculosis causing a severe gastrointestinal bleeding. Report of one case
\end{abstract}

We report a 27 years old woman presenting with hematochezia. An upper endoscopy and colonoscopy did not reveal the bleeding site. Due to persistence of bleeding, the patient was operated, finding a fibrinous secretion surrounding bowel loops and a hyper vascularized concentric papular lesion. The involved bowel loop was excised. The pathological study of the surgical piece revealed intestinal tuberculosis. The patient started treatment for extra pulmonary tuberculosis.

Key words: Intestinal tuberculosis, bleeding, hematochezia.

\section{Resumen}

Reportamos el caso de una paciente de 27 años con tuberculosis extrapulmonar gastrointestinal que se manifestó clínicamente como una hemorragia digestiva baja masiva exanguinante requiriendo cirugía de urgencia, donde se realizó laparotomía exploradora con entero y colonoscopia intraoperatoria localizando lesión a nivel de intestino delgado, resecando segmento comprometido con anastomosis primaria, evolución satisfactoria sin complicaciones postoperatorias.

Palabras clave: Tuberculosis intestinal, hemorragia digestiva, enteroscopia intraoperatoria.

\section{Introducción}

La tuberculosis (TBC) es una patología infecciosa originado por el Mycobacterium tuberculosis, su morbilidad y mortalidad va en disminución, pero se ha descrito un aumento del número de casos debido principalmente al aumento en la incidencia del síndrome de inmunodeficiencia adquirida (SIDA) ${ }^{1,2}$. Cerca del $20 \%$ de las TBC son extrapulmonares, aumentando considerablemente en pacientes con SIDA. Más del 5\% de las TBC tienen afección abdominal, siendo el tracto gastrointestinal el más frecuentemente comprometido ${ }^{3,4}$. Las complicaciones más frecuentes de la TBC intestinal son la obstrucción intestinal, perforaciones, fístulas y la hemorragia digestiva masiva, siendo esta última poco frecuente 5 .

No existen publicaciones nacionales que docu-

*Recibido el 16 de Mayo de 2010 y aceptado para publicación el 19 de Junio de 2010.

Correspondencia: Dr. Ricardo Villalón C.

Gran Avenida 3204, Santiago, Chile.

E-mail: revc73@gmail.com 
menten un caso de hemorragia digestiva masiva exanguinante de origen tuberculoso intestinal.

Se reporta el caso de una paciente que presentó úlcera tuberculosa intestinal, manifiestada como una hemorragia digestiva baja masiva que requirió tratamiento quirúrgico de urgencia.

\section{Caso clínico}

Mujer de 27 años, sin antecedentes mórbidos, consulta por cuadro de dos semanas de astenia, adinamia, anorexia, fiebre, lipotimia en seis episodios y distensión abdominal, asociándose a hematoquezia de abundante cuantía esporádica. Ingresa al Servicio de Urgencia del Complejo Asistencial Barros Luco Trudeau por aumento de intensidad de los síntomas y por sangrado por ano fresco no defecatorio.

A su ingreso se constata compromiso hemodinámico, subfebril, sudorosa y pálida. En el examen físico destaca abdomen depresible, sensible en flanco izquierdo, sin signos de irritación peritoneal y sangre fresca por ano. En exámenes de ingreso destaca hematocrito de $24 \%$, hemoglobina de $8 \mathrm{mg} /$ dl con recuento leucocitario normal, acidosis metabólica compensada, protrombinemia de $61 \%$ y PCR dentro de límites normales. Se inicia reanimación aportándose volumen y transfundiéndose 4 U GR, logrando estabilización temporal permitiendo el estudio endoscópico de urgencia con panendoscopia alta que no evidencia sangrado, y colonoscopia con ileoscopia que no identifica lesiones sangrantes en recto ni colon pero demuestra abundante sangre fresca en íleon terminal.

Persiste sangrado fresco por ano, compromiso hemodinámico, no logrando su estabilización. Frente a la imposibilidad de contar con arteriografía de urgencia se decide su cirugía. Se realiza laparotomía media supra e infraumbilical, donde destaca una tenue secreción fibrinosa sobre asas intestinales y lesiones papulares blanquecinas múltiples sobre asas de intestino delgado que al retirarlas digitalmente dejan ver una lesión papular hipervascularizada concéntrica (Figura 1). Se realiza colonoscopia y enteroscopia intraoperatoria, guiado a través de la laparotomía sin la necesidad de realizar enterotomías. No se encontraron lesiones en mucosa colorrectal ni en mucosa relacionada a lesiones intestinales descritas previamente, pero a $180 \mathrm{~cm}$, proximales a la válvula ileocecal, se observa úlcera circunferencial con sangrado activo (Figura 2), no siendo posible su control por vía endoscópica. Se decide resección segmentaria del asa intestinal comprometida (Figura 3) con anastomosis manual primaria término-terminal. Realiza su postoperatorio en Unidad de Paciente Crítico donde evoluciona favorablemente, extubándose en las primeras 24 horas postoperatorias, sin requerimientos de oxígeno, ni drogas vasoactivas. Inicia tránsito intestinal a las 48 horas postoperatorias, buena tolerancia a alimentación oral y es dada de alta al $5^{\circ}$ día postoperatorio sin complicaciones médicas o quirúrgicas.

El estudio diferido destaca antigenemia para citomegalovirus negativo, anticuerpos antinucleares negativo e hipocomplementemia con C3 de $54 \mathrm{mg} /$ dL y C4 de $7 \mathrm{mg} / \mathrm{dL}$. La biopsia diferida muestra tejido fibroadiposo con proceso inflamatorio crónico con granulomas caseificantes y células gigantes multinucleares de Langerhans compatibles con tuberculosis intestinal (Figura 4). La PCR de biopsia para Mycobacterium tuberculosis fue positiva. Derivada a unidad de infecciosos donde se completó estudio con anticuerpos para $\mathrm{VIH}$, radiografía de tórax y baciloscopias, las que fueron negativas, e inició tratamiento para TBC extrapulmonar.

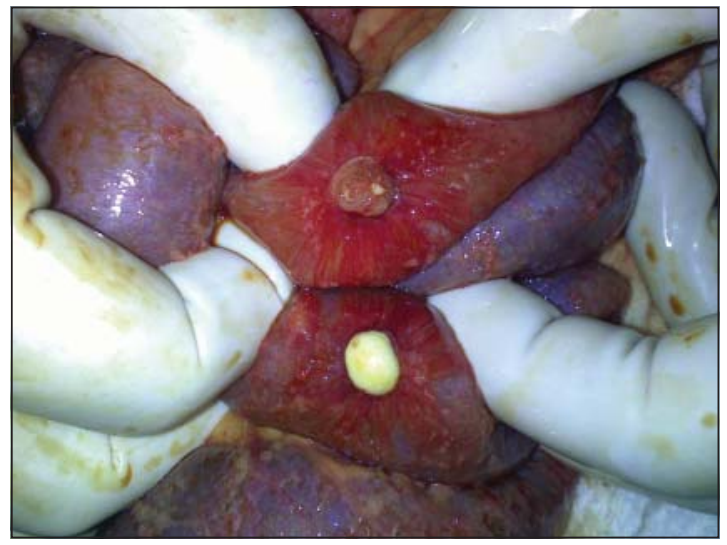

Figura 1. Lesión papular.

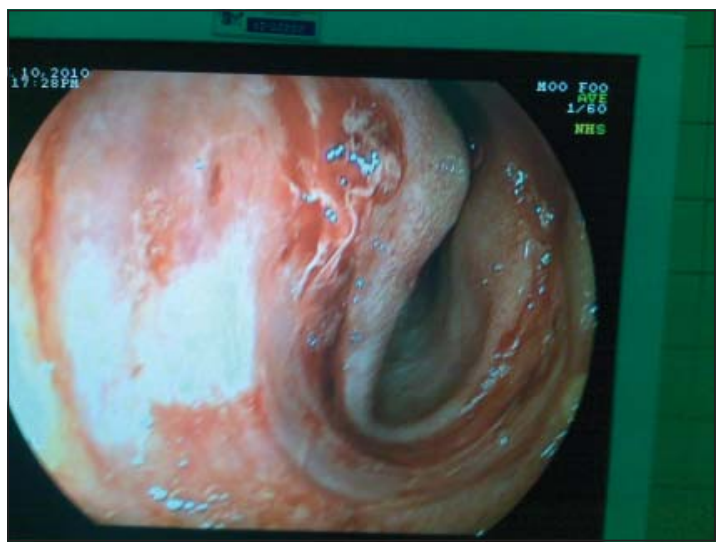

Figura 2. Úlcera. 


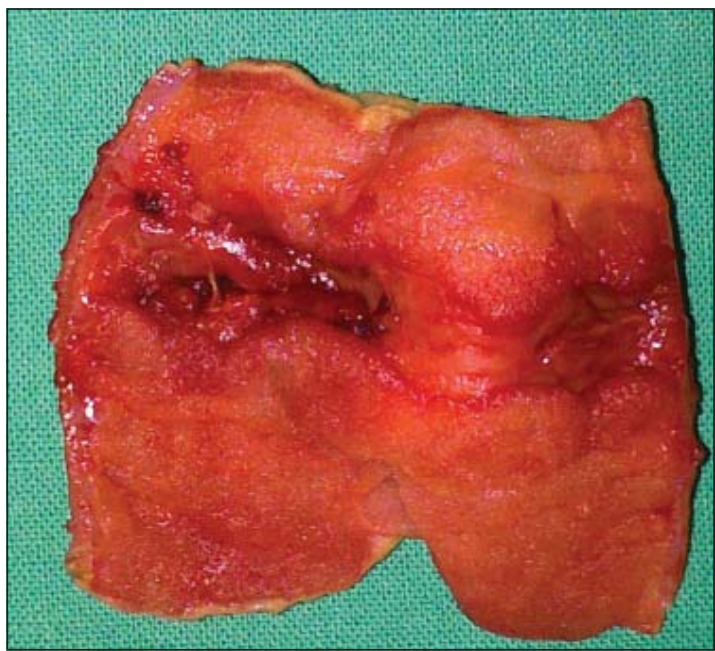

Figura 3. Asa resecada.

\section{Discusión}

Se cree que la disminución en las últimas décadas de la TBC gastrointestinal en pacientes inmunocompetentes, se debe al desarrollo de nuevas terapias antituberculosas y al consumo de leche pasteurizada. Pero en los últimos años esta variedad se ha incrementado debido a factores como la mejoría en los métodos diagnósticos y el mayor número de pacientes inmunocomprometidos ${ }^{6,7}$.

El compromiso de la TBC del tubo digestivo se alcanza por diseminación hematógena o linfática durante la fase de bacteremia o por la deglución del bacilo en pacientes con TBC pulmonar activa. Hoy en día es muy remoto el consumo de leche contaminada con la cepa Bovis del bacilo, dada la pausterización de la leche ${ }^{1}$.

La afección digestiva tiene distintas formas de presentación, siendo el compromiso intestinal el más frecuente, seguido por el peritoneal y la linfadenitis mesentérica. Afectando a pacientes jóvenes generalmente en la tercera o cuarta década de la vida sin diferencia por sexo ${ }^{8}$. En nuestra paciente se presentó con manifestación intestinal y peritoneal (Figuras 1 y 2).

El compromiso en conjunto pulmonar y digestivo, se puede presentar desde un $5 \%$ a un $38 \%$ de los pacientes asociado en general a condiciones socioeconómicas adversas ${ }^{9}$. No presente en nuestro caso.

En la TBC intestinal la región ileocecal se afecta en un $90 \%$ de los $\operatorname{casos}^{10}$. Se puede presentar en tres patrones: ulcerativo, hiperplásico y esclerótico o estenótico. La forma ulcerativa es la más frecuente ubicándose en íleon y yeyuno y se caracteriza por

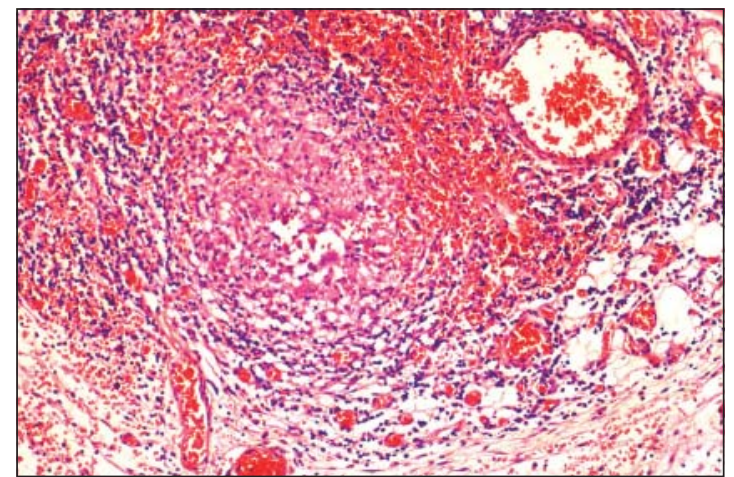

Figura 4. Histología TBC. múltiples lesiones ulcerativas pequeñas con mucosa aledaña sana ${ }^{1}$. En nuestro caso se presentó como una lesión única ulcerativa circunferencial.

La presentación del cuadro clínico suele ser de manera insidiosa, pero puede presentarse de manera aguda en un $20 \%$ a $36 \%$ principalmente por complicaciones, siendo el sangrado digestivo poco frecuente ${ }^{1}$.

La hemorragia digestiva baja masiva (HDBM) es el sangrado digestivo distal al ángulo de Treitz, que requiere de al menos 2 unidades de glóbulos rojos para mantener estabilidad hemodinámica, entre un $10 \%$ y $45 \%$ de estas HDBM requieren cirugía de urgencia ya sea por persistencia de sangrado o por ser exanguinante ${ }^{11-13}$.

La hemorragia digestiva originada en el intestino delgado constituye el $2 \%$ al $10 \%$ de todas las hemorragias digestivas, siendo lo más frecuente las malformaciones vasculares y los tumores ${ }^{14,15}$.

HDBM exanguinante es aquella en que a pesar del aporte de fluidos y sangre no es posible estabilizar hemodinámicamente al paciente, persistiendo el sangrado. Es una emergencia y un reto para todo el equipo médico de urgencia. En general no permite pérdida de tiempo en técnicas diagnósticas que requieren de mayor complejidad y que por lo general no se encuentran disponibles en los servicios de urgencia.

Frente a esta catastrófica situación la cirugía es la única alternativa terapéutica posible, con la enorme limitación que en la mayoría de los casos no tenemos la localización exacta del sangrado. En nuestro caso específico pudimos contar con exámenes endoscópicos preoperatorios que descartaban el origen alto y colorrectal, dejando el segmento de intestino 
delgado como eventual origen. La enteroscopia y colonoscopia intraoperatoria es considerada un método eficaz desde el punto diagnóstico y terapéutico en pacientes con hemorragia digestiva de causa no precisada, localizando el sitio de sangrado. Puede utilizarse una o más enterotomías para la introducción del endoscopio ${ }^{16-18}$. En nuestra paciente pudimos contar con entero y colonoscopia intraoperatoria no realizando enterotomías, lo que permitió localizar el sitio exacto de sangrado realizando una cirugía menos invasiva, sin complicaciones postoperatorias.

\section{Referencias}

1. Maroto N, Ponce M. Tuberculosis y aparato digestivo. Gastroenterol Hepatol 2003; 26: 34-41.

2. Raviglione M, Snidar D, Kochi A. Global epidemiology of tuberculosis. Morbidity and mortality of a worldwide epidemic. JAMA 1995; 273: 220-226.

3. Horvath K, Whelan R. Intestinal tuberculosis: return of an old disease. Am J Gastroenterol 1998; 93: 692-696.

4. Swroop VS. Intestinal tuberculosis. Am J Gastroenterol 1999; 94: 1982.

5. Ha HK, Ko GY, Yu ES, Yoon K, Hong WS, Kim HR, et al. Intestinal tuberculosis with abdominal complications: radiologic and pathologic features. Abdom Imaging 1999; 24: 32-38

6. Velásquez J, Szigethi M, Panace R, Morales R, Márquez $\mathrm{S}$, Pefaur J, y cols. Micobacteriosis hepato-esplénica, forma inusual de probable tuberculosis extrapulmonar. Caso clínico y revisión bibliográfica. Rev Chil Infect 2007; 24: 59-62.

7. Mackenna M, Mccray E, Onorato I. The epidemiology of tuberculosis among foreignborn persons in the United
States, 1986 to 1993. N Engl J Med 1995; 332: 10711076.

8. Faylona J, Chung S. Abdominal tuberculosis revisited. Ann Coll Surg Hong Kong 1999; 3: 65-70.

9. Baehr PH, McDonald GB. Esophageal infections: risk factors, presentation, diagnosis and treatment. Gastroenterology 1994; 106: 509-532.

10. Demir K, Okten A, Kaymakoglu S, Dincer D, Besisik $\mathrm{F}$, Cevikbas U, et al. Tuberculous peritonitis- reports of 26 cases, detailing diagnostic and therapeutic problems. Eur J Gastroenterol Hepatol 2001; 13: 581-585.

11. Browder W, Cerise EJ, Litwin MS. Impact of emergency angiography in massive lower gastrointestinal bleeding. Ann Surg 1986; 204: 530-536.

12. Bannura G. Hemorragia digestiva baja. En Llorens $P$, Nakamura K. Diagnóstico y tratamiento de las enfermedades del colon. JICA. Ed. Abaco; Tokio, Japón. 1990; 56-72.

13. Vernava AM, Moore BA, Longo WE, Johnson FE. Lower gastrointestinal bleeding. Dis Colon Rectum 1997; 40: 846-858.

14. Manning-Dimmitt LL, Dimmitt SG, Wilson GR. Diagnosis of bleeding in adults. Am Fam Physician 2005; 71: 1339/1346.

15. Saperas E. Lower gastrointestinal bleeding: The greatun-known. Gastro enterol Hepatol 2007; 30: 93-100.

16. Mathus-Vliegen EM, Tytgat GN. Intraoperative endoscopy: Technique, indications, and results. Gastrointest Endosc 1986; 32: 381-384.

17. Mihara Y, Kubota K, Nagata H, Takagi K, Horie T, Oda $\mathrm{N}$, et al. Total intraoperative enteroscopy using a colonoscope for detecting the bleeding point. Hepatogastroenterology 2004; 51: 1401-1403.

18. Whelan RL, Buls JG, Goldberg SM, Rothenberger DA. Intrao-perative endoscopy. University of Minnesota experience. AmSurg 1989; 55: 281-286. 